\title{
Finite-difference modeling with variable grid-size and adaptive time-step in porous media
}

\author{
Xinxin Liu $\cdot$ Xingyao Yin $\cdot$ Guochen Wu
}

Received: 29 July 2013/ Accepted: 16 December 2013/Published online: 18 January 2014

(C) The Seismological Society of China, Institute of Geophysics, China Earthquake Administration and Springer-Verlag Berlin Heidelberg 2014

\begin{abstract}
Forward modeling of elastic wave propagation in porous media has great importance for understanding and interpreting the influences of rock properties on characteristics of seismic wavefield. However, the finite-difference forward-modeling method is usually implemented with global spatial grid-size and time-step; it consumes large amounts of computational cost when small-scaled oil/ gas-bearing structures or large velocity-contrast exist underground. To overcome this handicap, combined with variable grid-size and time-step, this paper developed a staggered-grid finite-difference scheme for elastic wave modeling in porous media. Variable finite-difference coefficients and wavefield interpolation were used to realize the transition of wave propagation between regions of different grid-size. The accuracy and efficiency of the algorithm were shown by numerical examples. The proposed method is advanced with low computational cost in elastic wave simulation for heterogeneous oil/gas reservoirs.
\end{abstract}

Keywords Staggered-grid finite-difference scheme . Variable grid-size $\cdot$ Variable time-step $\cdot$ Porous media

\section{Introduction}

Heterogeneous reservoirs, such as fractured or cavernous carbonate reservoirs, tight sandstone reservoirs, etc., have gradually become the hotspots of seismic exploration. These reservoirs exhibit two-phase features caused by the

X. Liu $(\bowtie) \cdot X$. Yin · G. Wu

College of Geosciences, China University of Petroleum,

Qingdao 266580, China

e-mail: xxinl@126.com fluid in pores, cracks or fractures, and multi-scale features caused by complex structures and strong heterogeneous reservoirs. Both of these features should be considered in the explanation and prediction for heterogeneous oil/gas reservoirs. The numerical modeling of seismic waves in heterogeneous and porous reservoirs is an important tool for seismic interpretation (Wenzlau and Müller 2009).

Biot $(1956,1962)$ established the dynamic equations of elastic wave propagation in saturated porous media for the first time and predicted the existence of two types of longitudinal waves and one type of shear wave. The Biot theory has been regarded as a basis for wave propagation analysis in porous media. Since the analytical solutions of wave equations are difficult to obtain, different numerical methods are proposed by many authors. Finite-difference methods have been widely used because of low computational cost, ease of implementation, and strong adaptability. Staggered-grid finite difference (SGFD) method can further improve the precision and suppress dispersion without additional computational cost (Wu 2006). Hassanzadeh (1991) first solved the low-frequency Biot poroacoustic equations using a second-order finite-difference (FD) scheme. After that, FD method was extended and improved to solve poroelastic wave equations (Zhu and McMechan 1991; Dai et al. 1995; Carcione and QuirogaGoode 1995; Carcione and Helle 1999; Santos et al. 2004; Wenzlau and Müller 2009; Sun and Yin 2011).

SGFD method uses regular grid to discretize seismic models and may lead to numerical dispersion until spatial grid-size is small enough. That means, for low velocity layers or heterogeneous bodies (such as multi-scale structures or interfaces with high contrast between physical parameters, etc.) surrounded by high-velocity homogeneous media, grid-size should be small enough to assure sufficient sampling with small time-step according to the FD stability 
condition. However, simulation with this small grid-size and time-step leads to oversampling in surrounding high-velocity homogeneous media and results in additional computational cost. To achieve the balance of spatial sampling in geological structures at different scales, Moczo (1989) first proposed the idea of varied grid-size to decrease computational cost. This idea have been improved for elastic wave simulation (Jastram and Tessmer 1994; Pitarka 1999; Hayashi et al. 2001; Wang et al. 2001), but the flexibility and accuracy of these methods are insufficient since the spatial grid-size variation is limited to single direction and grid-size ratio is restricted under three. The variable-grid FD schemes then have been developed with grid-size variation in both spatial dimensions (Zhu et al. 2007; Li et al. 2008; Guilbot 2008). However, the computational efficiency is still not good enough because of unchanged time step.

The local variable time-step methods were proposed to solve this problem (Falk et al. 1998; Tessmer 2000; Kang and Baag 2004; Huang and Dong 2009; Sun and Yin 2011). These methods solve the temporal oversampling problem with detailed description of local structures and refined temporal effectiveness. In this paper, SGFD method with variable spatial grid-size and variable timestep is extended to simulate elastic wave propagation in porous media. Small grid-size and time-step is used for heterogeneous bodies such as fractured or cavernous bodies, regions with high physical parameter contrasts, etc., while big grid-size and time-step are used for surrounding homogeneous media. Different FD coefficients and wavefield interpolation are used to realize the transition of wave propagation between regions discretized by different spatial grid-size.

\section{Methodology}

According to Biot's theory (Biot 1956), combined with Newtonian dynamic equations and Darcy's law (Zhang, et al. 2004), the stress and velocity equations of wave propagation in fluid-saturated, statistically isotropic, nonuniform porous media can be derived as

$$
\begin{aligned}
& \dot{\sigma}_{i j}=N\left(v_{i, j}+v_{j, i}\right)+\delta_{i j}\left(A v_{k, k}+Q V_{k, k}\right) \\
& \dot{S}=R\left(V_{i, j}+V_{j, i}\right)+Q\left(v_{i, i}+v_{j, j}\right) \\
& \dot{v}_{i}=A_{22} \sigma_{i j, j}-A_{12} S_{, i}-b\left(A_{12}+A_{22}\right)\left(v_{i}-V_{i}\right) \\
& \dot{V}_{i}=-A_{12} \sigma_{i j, j}+A_{11} S_{, i}+b\left(A_{11}+A_{12}\right)\left(v_{i}-V_{i}\right)
\end{aligned}
$$

where $A, N, Q, R$ are elastic parameters of porous media; $\delta_{i j}$ is Kronecker function. $\sigma_{i j}$ is solid stress, $S$ is fluid effective strain; $A_{i j}=\rho_{i j}\left(\rho_{11} \rho_{22}-\rho_{12}^{2}\right)^{-1}, \rho_{i j}$ is mass parameters and are related to the mass parameters of solid and fluid per unit volume, $\rho_{1}$ and $\rho_{2}: \quad \rho_{11}+\rho_{12}=(1-\phi) \rho_{1}$, $\rho_{22}+\rho_{12}=\phi \rho_{2} ; \phi$ is porosity; $b$ is dissipation parameter.

\subsection{Staggered-grid finite-difference scheme based on PML boundary condition}

Discretize the model into numbers of grids with certain grid-size, define model parameters and wavefield variables in the main grids and staggered grids which are staggered by half the grid spacing, as shown in Fig. 1 .

The FD scheme is also staggered in time. Let $k, k \pm$, $k \pm 1$ represent time $t, t \pm \frac{1}{2} \Delta t, t \pm \Delta t$, respectively, substitute the FD formation of first-order time derivation into Eq. (1), we obtain the SGFD equations (take Eq. (1c)) for example and let $i=j=1$ ) as

$$
\begin{aligned}
v_{x}^{k+}= & v_{x}^{k-}+\Delta t\left[A_{22} L_{x}^{+} \sigma_{x x}^{k}+L_{z}^{-} \sigma_{x z}^{k}-A_{12} L_{x}^{+} S^{k}\right. \\
& \left.-b\left(A_{12}+A_{22}\right)\left(v_{x}^{k-}-V_{x}^{k-}\right)\right]
\end{aligned}
$$

where $L_{x}^{+}, L_{x}^{-}$denote forward and backward finite-difference operators along $x$ direction, respectively.

The $(2 N)$ th-order SGFD operator derived by Taylor series expansion (Wu 2006) is given by

$$
\begin{aligned}
\frac{\partial v_{x}}{\partial x}= & \frac{1}{\Delta x} \sum_{i=1}^{N} C_{i}^{(N)}\left\{v_{x}\left[x+\frac{\Delta x}{2}(2 i-1)\right]\right. \\
& \left.-v_{x}\left[x-\frac{\Delta x}{2}(2 i-1)\right]\right\}+O\left(\Delta x^{2 N}\right)
\end{aligned}
$$

where $C_{i}^{(N)}(i=1,2, \cdots, N)$ are FD coefficients determined by the following equations:

$\boldsymbol{F} \boldsymbol{C}^{(\mathbf{N})}=\boldsymbol{E}$

where $\quad F_{i j}=(2 j-1)^{2 i-1}, \quad i, j=1,2, \cdots, N, \quad \boldsymbol{E}=(1,0$, $\cdots, 0)^{T}$. Combined with the definition of grids and model parameters, the forward and backward SGFD operators are

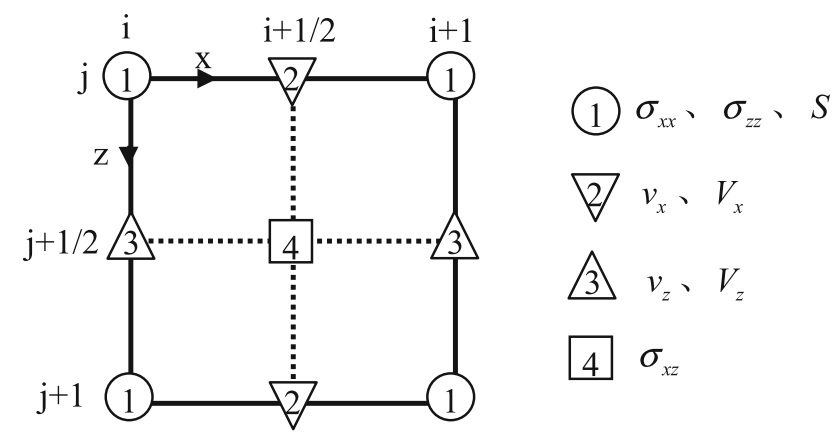

Fig. 1 Definition of the main grids and staggered grids used in SGFD modeling 


$$
\begin{aligned}
L_{x}^{ \pm} v_{x}= & \frac{1}{\Delta x} \sum_{i=1}^{N} C_{i}^{(N)}\left\{v_{x}\left[x+\frac{\Delta x}{2}(2 i+1) \pm \frac{\Delta x}{2}\right]\right. \\
& \left.-v_{x}\left[x-\frac{\Delta x}{2}(2 i+1) \pm \frac{\Delta x}{2}\right]\right\}
\end{aligned}
$$

Truncating the unbounded media with artificial boundaries will introduce spurious reflections. The perfectly matched layer (PML) absorbs outgoing waves by imposing artificial absorptive layers outside the regular media ( $\mathrm{He}$ 2008; Hastings et al. 1996). In this paper, the SGFD formula with PML was derived by modifying Eq. (1) with complex coordinates.

In frequency domain, introduce a complex factor by which the Cartesian coordinates are extended to complex coordinates (He 2008)

$\tilde{n}=\int_{0}^{n} s_{n}\left(n^{\prime}\right) d n^{\prime}, s_{n}(n)=1+d_{n}(n) / i \omega$

where $n$ represents spatial coordinates; $i=\sqrt{-1} ; d_{n}$ is attenuation factor independent of frequency, $d_{n}>0$ inside PML and $d_{n}=0$ outside PML. According to Eq. (6), the operator $\partial / \partial n$ can be expressed in terms of the complex coordinates

$\frac{\partial}{\tilde{n}}=\frac{1}{\mathrm{~s}_{n}} \cdot \frac{\partial}{n}$

where a time dependent term $n$ is implied. The PML formulation is to replace $n$ in Eq. (1) by the corresponding complex coordinate $\tilde{n}$. Take the velocity component along $x$ direction for example, we have

$$
\begin{aligned}
i \omega \tilde{v}_{x}= & A_{22}\left(\frac{1}{s_{x}} \frac{\partial \tilde{\sigma}_{x x}}{\partial x}+\frac{1}{s_{z}} \frac{\partial \tilde{\sigma}_{x z}}{\partial z}\right)-A_{12} \frac{1}{s_{x}} \frac{\partial \tilde{S}}{\partial x} \\
& -b\left(A_{12}+A_{22}\right)\left(\tilde{v}_{x}-\tilde{V}_{x}\right)
\end{aligned}
$$

In order to simplify the PML equations, the variables are split along spatial coordinates

$\tilde{v}_{x}=\tilde{v}_{x}^{x}+\tilde{v}_{x}^{z}$

then Eq. (8) can be split into two equations

$$
\begin{aligned}
\left(i \omega+d_{x}\right) \tilde{v}_{x}^{x}= & A_{22} \frac{\partial \tilde{\sigma}_{x x}}{\partial x}-A_{12} \frac{\partial \tilde{S}}{\partial x} \\
& -\left(1+d_{x} / i \omega\right) b\left(A_{12}+A_{22}\right)\left(\tilde{v}_{x}^{x}-\tilde{V}_{x}^{x}\right) \\
\left(i \omega+d_{z}\right) \tilde{v}_{x}^{z}= & A_{22} \frac{\partial \tilde{\sigma}_{x z}}{\partial z}-\left(1+d_{z} / i \omega\right) b\left(A_{12}+A_{22}\right) \\
& \left(\tilde{v}_{x}^{z}-\tilde{V}_{x}^{z}\right)
\end{aligned}
$$

Transform the equations back to time-space domain and substitute the SGFD operators of first-order time and spatial derivatives into, we obtain

$$
\begin{aligned}
\left(v_{x}^{x}\right)^{k+1}= & g_{x}\left(v_{x}^{x}\right)^{k}+h_{x}\left[A_{22} L_{x}^{+} \sigma_{x x}^{k+}-A_{12} L_{x}^{+} S^{k+}\right] \\
& +h_{x} b\left(A_{12}+A_{22}\right)\left[\left(v_{x}^{x}\right)^{k}-\left(V_{x}^{x}\right)^{k}-D_{x}\right] \\
\left(v_{x}^{z}\right)^{k+1}= & g_{z}\left(v_{x}^{z}\right)^{k}+h_{z} A_{22} L_{z}^{-} \sigma_{x z}^{k+} \\
& +h_{z} b\left(A_{12}+A_{22}\right)\left[\left(v_{x}^{z}\right)^{k}-\left(V_{x}^{z}\right)^{k}-D_{z}\right]
\end{aligned}
$$

where $\quad D_{n}=d_{n} \int_{0}^{k}\left(v_{x}^{n}-V_{x}^{n}\right) d \tau, \quad g_{n}=\left(2-d_{n} \Delta t\right)$ $\left(2+d_{n} \Delta t\right)^{-1}$ and $h_{n}=2 \Delta t\left(2+d_{n} \Delta t\right)^{-1}$. Similar equations can be obtained for other velocity and stress components. It is obvious that outside PML, the combination of Eqs. (9) and (11) degenerate into Eq. (2), which means the introduction of PML do not change the wavefields outside PML.

\subsection{Staggered-grid finite-difference scheme with variable grid-size and time-step}

Geological bodies at different scales are discretized with different grid-size. Small grid-size is used for small-scaled structures or fractured bodies and big grid-size is used for their surrounding media. There are two methods to deal with transition zone between small and big grid-size regions: interpolation method and variable coefficient method (Sun and Ding 2012). This paper introduces variable coefficient method which is of higher simulation precision.

Let $\Delta x$ and $\Delta z$ represent small grid-size along $x$ and $z$ direction, respectively, $M \Delta x, M \Delta z\left(M \in N_{+}\right.$is the ratio of big and small grid-size) represent big grid-size along $x$ and $z$ direction, respectively. High-order SGFD operators are used in both big and small grid-size regions. In transition zone (gray shaded area in Fig. 2), the FD coefficients should be recalculated to maintain the symmetry of SGFD operators and suppress artificial reflections arises by the interface of big and small grid-size region.

Take sixth-order SGFD operator with $M=3$ for example, for first-order spatial derivations along $x$ direction, grid nodes used to calculate the SGFD operators are shown in Fig. 3, the black stars represent the computing nodes (center of the SGFD operators), black circles represent nodes involved for the calculation. Formulations and coefficients of the FD

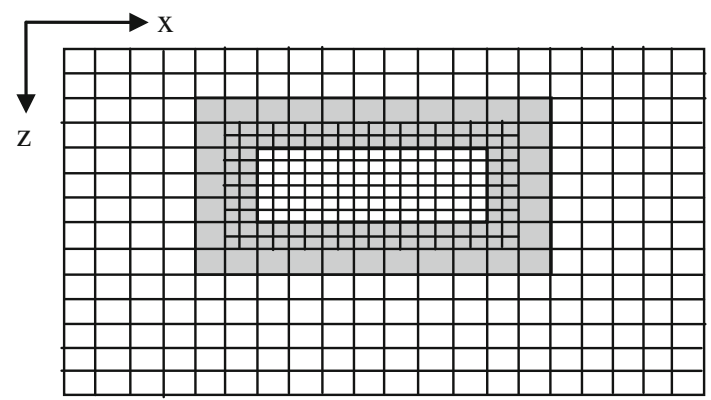

Fig. 2 Configuration of model discretion with spatial grids (gray shaded area represents the transition zone) 


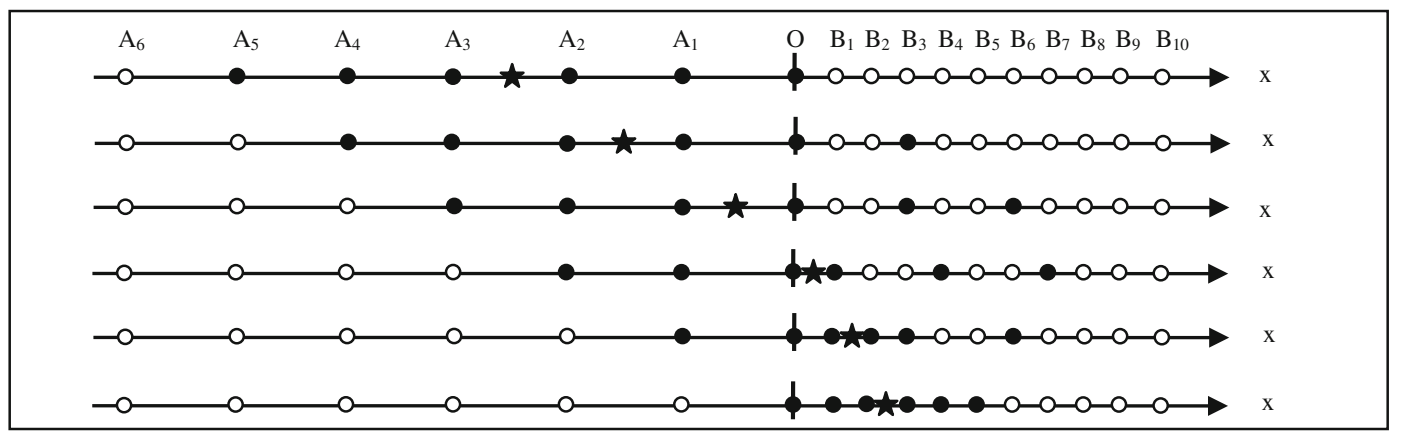

Fig. 3 Formulations of FD operators in transition zone

operator of three nodes in the left of the interface (between big grid-size region and small grid-size region) remain unchanged, while the nodes involved for calculation should be symmetric about the center node. Formulations of the FD operator at two nodes in the right of the interface becomes

$$
\begin{aligned}
\frac{\partial v_{x}}{\partial x}= & \frac{1}{\Delta x} \sum_{i=1}^{n} \bar{C}_{i}^{(N)}\left\{v_{x}\left[x+\frac{\Delta x}{2}(2 i-1)\right]-v_{x}\left[x-\frac{\Delta x}{2}(2 i-1)\right]\right\} \\
& +\frac{1}{M \Delta x} \sum_{i=1+n}^{N} \bar{C}_{i}^{(N)}\left\{v_{x}\left[x+\frac{\Delta x}{2}(2 n-1)+(i-n) M \Delta x\right]\right. \\
& \left.-v_{x}\left[x-\frac{\Delta x}{2}(2 n-1)-(i-n) M \Delta x\right]\right\}+O\left(\Delta x^{2 N}\right)
\end{aligned}
$$

$\bar{C}_{i}^{(N)}$ is FD coefficients determined by

$\boldsymbol{F} \boldsymbol{C}^{(\mathbf{N})}=\boldsymbol{E}$

where $\quad \bar{F}_{i j}=\left\{\begin{array}{ll}(2 j-1)^{2 i-1} & j<=n \\ {[(2 n-1)+2 M(j-n)]^{2 i-1}} & n<j<=N,\end{array}\right.$, $\mathbf{E}=(1,0, \cdots, 0)^{T}$.

The stability condition for elastic-wave propagation holds also for the poroelastic case (Wenzlau and Müller 2009). The stability of the numerical scheme with $(2 N)$ thorder spatial and second-order time FD operator for elasticwave propagation (Huang and Dong 2009) is

$\Delta t^{\prime}\left(\frac{1}{\Delta x^{\prime 2}}+\frac{1}{\Delta z^{\prime 2}}\right)^{2} \leq \frac{1}{V_{P, \max }}\left[\sum_{j=1}^{N} C_{j}^{(N)}(-1)^{j-1}\right]^{-1}$

where $V_{P, \max }$ is the maximum $\mathrm{P}$-wave velocity in big and small grid-size region. Equation (14) must be satisfied in big and small grid-size region, respectively. Variable spatial grid-size method would not make the stability condition more rigid (Huang and Dong 2009).

To satisfy the stability condition, time-step in small grid-size region is adaptively defined according to gridsize using Eq. (14). Let $\Delta t$ and $P \Delta t$ represent small and big time-step, respectively, first calculate wavefields using big grid-size $M \Delta x, M \Delta z$ and time-step $P \Delta t$, save the field variables of the transition zone, calculate the wavefields in small grid-size region at this moment using linear interpolation method, then do time stepping with time-step $\Delta t$ until time level $P \Delta t$ is reached. The transition of wavefield form big time-step to small time-step is achieved. The time-step ratio $P$ can be arbitrary positive integer adaptively determined by stability condition (14).

\section{Numerical examples}

\subsection{Homogeneous model of porous media}

We first examine the accuracy of the numerical solutions. Compare the elastic wave modeling results of variable grid-size and time-step SGFD (VSGFD) method with the analytical solution proposed by Dai et al. (1995) in a 2-D homogeneous poroelastic model. Model parameters are the same as those of the uniform model of Dai et al. (1995) (Table 1) and the damping coefficient $b$ is equal to zero. The size of model is $1000 \times 1000 \mathrm{~m}^{2}$. P-wave point source of Ricker time function with main frequency of $30 \mathrm{~Hz}$ locates at $(500 \mathrm{~m}, 500 \mathrm{~m})$. The big grid-size is $2 \mathrm{~m}$, timestep is $0.1 \mathrm{~ms}$; small grid-size is $0.4 \mathrm{~m}$, arranges form 50 to $600 \mathrm{~m}$ along $x$ direction and 200 to $400 \mathrm{~m}$ along $z$ direction, small time-step is $0.02 \mathrm{~ms}$.

The fast $\mathrm{P}$-wave velocity is $3210 \mathrm{~m} / \mathrm{s}$, slow $\mathrm{P}$-wave velocity is $1180 \mathrm{~m} / \mathrm{s}$, and the theoretical arrival time is 0.195 and $0.531 \mathrm{~s}$, respectively. The comparison of numerical results and analytical solutions (both have been normalized) are in Figs. 4 and 5. The arrival time, amplitude and frequency of numerical solutions fit those of analytical solutions very well. The VSGFD method is accurate enough to simulate the wave propagation in poroelastic media and was further tested in the following sections.

\subsection{Cavernous model of porous media}

The model contains two oil-bearing caves in the surrounding homogeneous media, the radius are 4 and $40 \mathrm{~m}$, respectively. The parameters are listed in Table 2. 
Table 1 Elastic parameters of homogeneous porous model

\begin{tabular}{llllllll}
\hline Parameters & $A\left(\mathrm{~kg} \cdot \mathrm{m}^{-1} \cdot \mathrm{s}^{-2}\right)$ & $N\left(\mathrm{~kg} \cdot \mathrm{m}^{-1} \cdot \mathrm{s}^{-2}\right)$ & $Q\left(\mathrm{~kg} \cdot \mathrm{m}^{-1} \cdot \mathrm{s}^{-2}\right)$ & $R\left(\mathrm{~kg} \cdot \mathrm{m}^{-1} \cdot \mathrm{s}^{-2}\right)$ & $\rho_{11}\left(\mathrm{~kg} / \mathrm{m}^{3}\right)$ & $\rho_{22}\left(\mathrm{~kg} / \mathrm{m}^{3}\right)$ & $\rho_{12}\left(\mathrm{~kg} / \mathrm{m}^{3}\right)$ \\
\hline Value & $6.652 \times 10^{9}$ & $6.84 \times 10^{9}$ & $0.953 \times 10^{9}$ & $0.331 \times 10^{9}$ & 2,167 & 191 & -83 \\
\hline
\end{tabular}

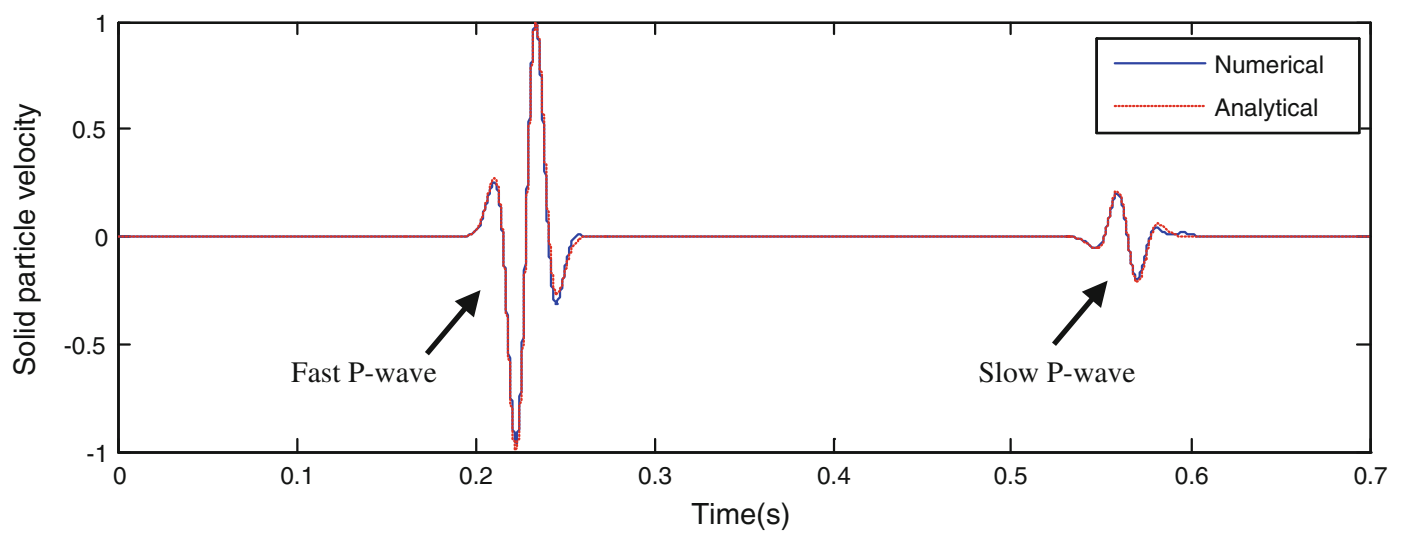

Fig. $4 \mathrm{Z}$ component wave record of solid phase [Receiver locates at (100 m, 20 m)]

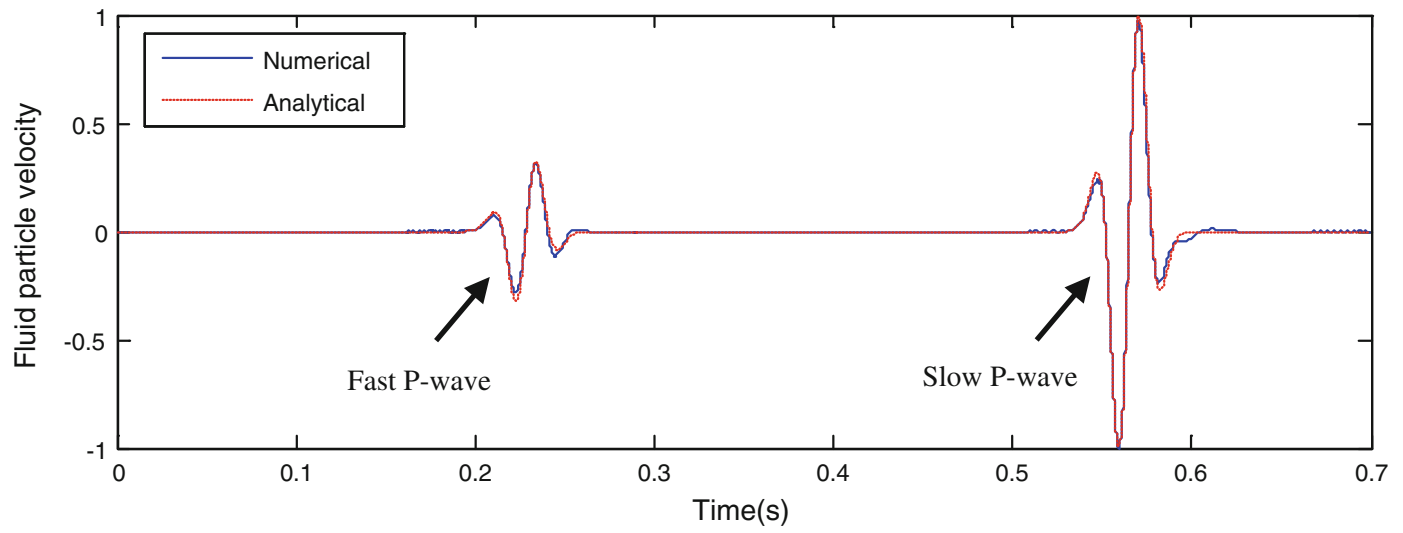

Fig. $5 \mathrm{Z}$ component wave record of fluid phase [Receiver locates at (100 m, $20 \mathrm{~m})$ ]

Table 2 Parameters of porous model with caves

\begin{tabular}{llllll}
\hline Model parameters & P-wave velocity $(\mathrm{m} / \mathrm{s})$ & S-wave velocity $(\mathrm{m} / \mathrm{s})$ & Density $\left(\mathrm{kg} / \mathrm{m}^{3}\right)$ & Porosity & Oil saturation \\
\hline Surrounding media & $5,210.26$ & $3,100.18$ & $2,569.10$ & 0.15 & 0.1 \\
Caves & 3,600 & 2,078 & 2,290 & 0.35 & 1.0 \\
\hline
\end{tabular}

The corresponding poroelastic parameters $A, N, Q, R$, $\rho_{11}, \rho_{22}$, and $\rho_{12}$ are calculated using rock physical theories with the method proposed by Geertsma and Smit (1961) and Berryman (1980).The big grid-size is $9 \mathrm{~m}$, time-step is $1 \mathrm{~ms}$; small grid-size is $3 \mathrm{~m}$ (inside dashed rectangle of Fig. 6). P-wave source with dominant frequency of $40 \mathrm{~Hz}$ locates at the middle of the model surface.

The modeling results of SGFD using big grid-size and time-step (BSGFD) could not simulate the reflected waves of the smaller cave (radius is $4 \mathrm{~m}$ ), results of VSGFD is similar to SSGFD and the reflection of the smaller cave can be seen at around $0.25 \mathrm{~ms}$. The computational time is 396.2, 1282.3, $7232.3 \mathrm{~s}$ by BSGFD, VSGFD, and SSGFD algorithm, respectively. The VSGFD method significantly improved the simulation efficiency while maintained the accuracy (Figs. 7, 8, 9).

\subsection{Low-velocity reservoir model of porous media}

The model contains a low-velocity reservoir in surrounding high-velocity layer, the model parameters are listed in

Table 3. The big grid-size is $10 \mathrm{~m}$, time-step is $1 \mathrm{~ms}$; small 


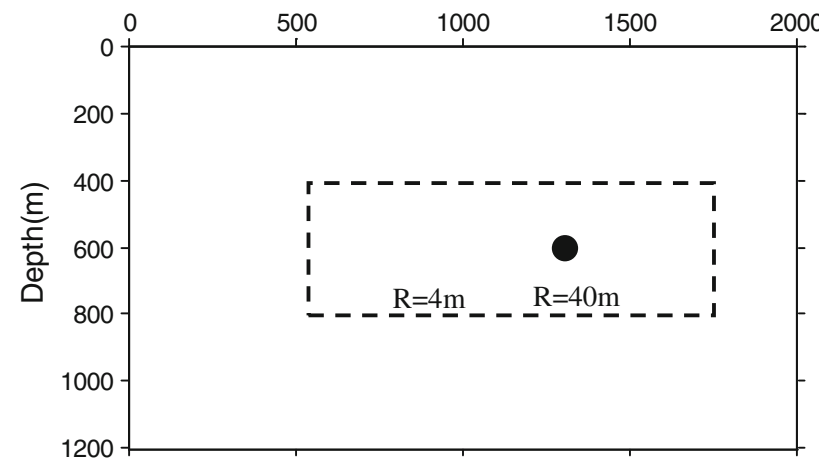

Fig. 6 Porous model with caves grid-size is $2 \mathrm{~m}$ (inside dashed rectangle of Fig. 10). Vertical force source with dominant frequency of $30 \mathrm{~Hz}$ locates at the middle of the model surface.

For the horizontal interface, reflection amplitude and arrival time simulated by BSGFD, VSGFD, SSGFD has little difference (Figs. 11, 12, 13, 14). Dispersion (can be seen after $0.65 \mathrm{~s}$ in Fig. 13) exists in the reflections of low-velocity reservoir simulated by BSGFD method. The SSGFD method can attenuate the dispersion but spent too much computational cost. The computational time is $55870.2 \mathrm{~s}$ while that spent by VSGFD method is $2991.6 \mathrm{~s}$, that means the simulation efficiency is much

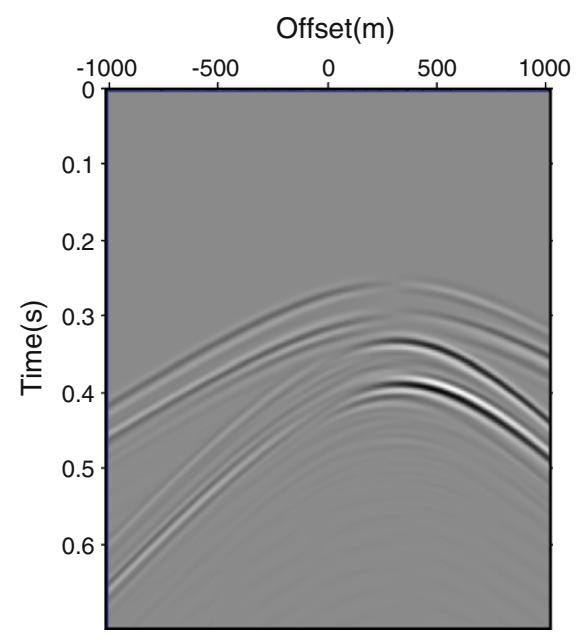

(a) BSGFD

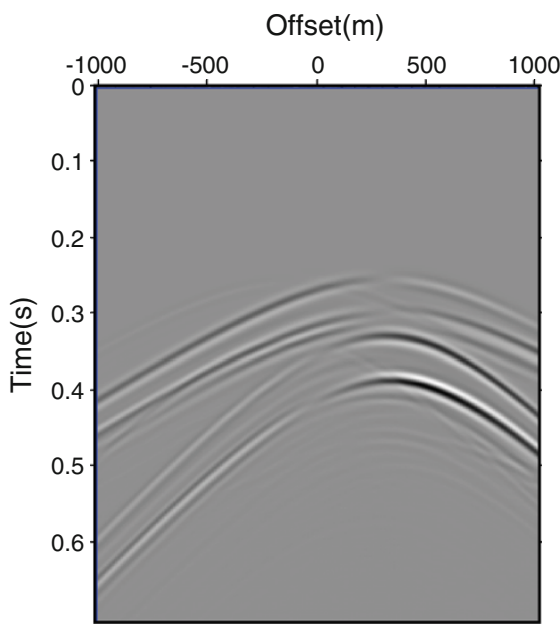

(b) VSGFD

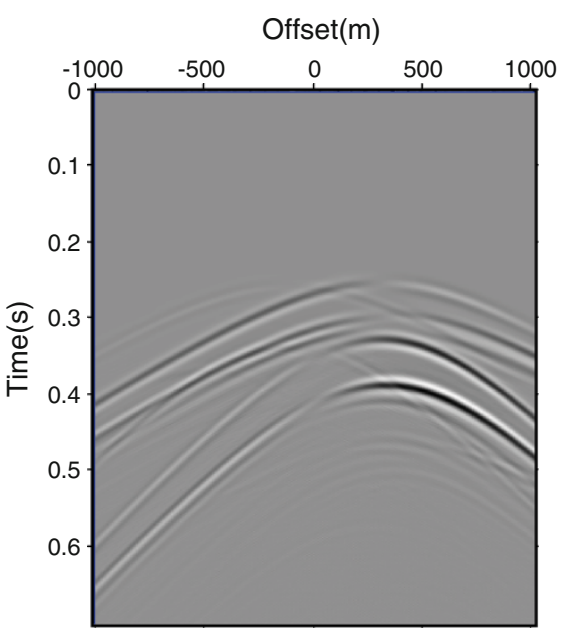

(c) SSGFD

Fig. $7 X$ component shot records of solid phase in porous model with caves

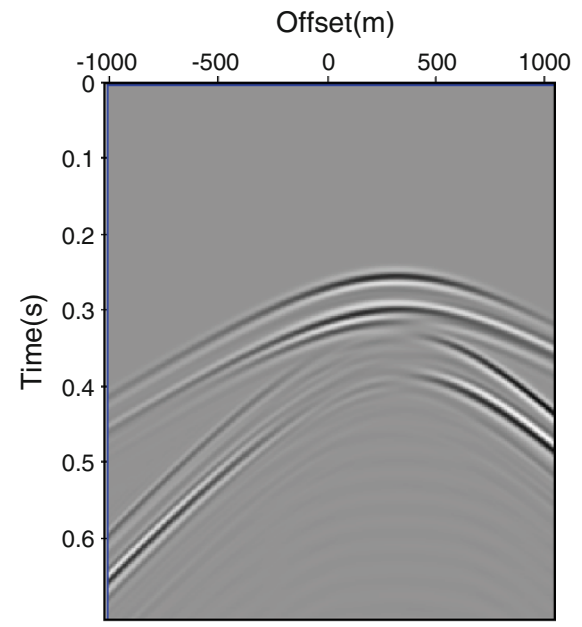

(a) BSGFD

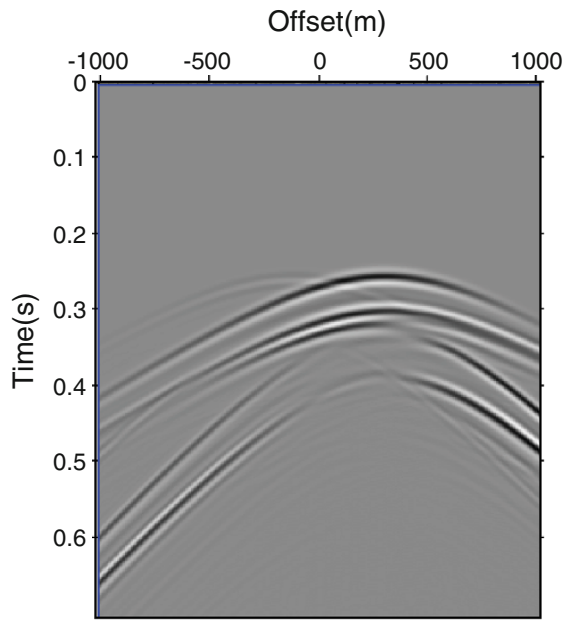

(b) VSGFD

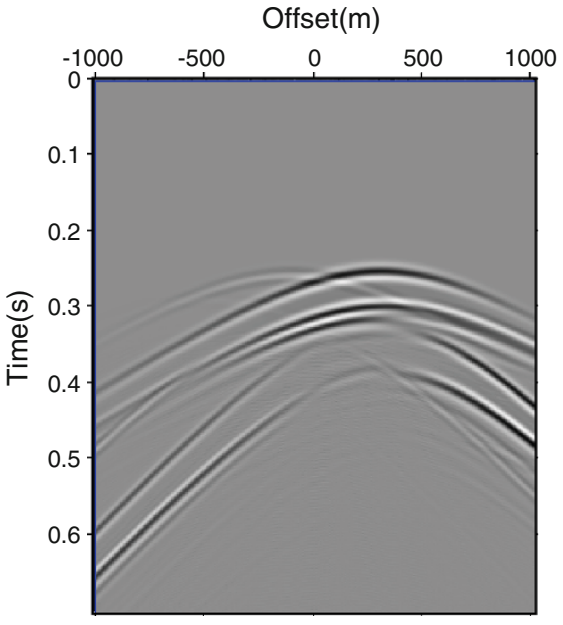

(c) SSGFD

Fig. $8 \mathrm{Z}$ component shot records of solid phase in porous model with caves (without direct wave) 


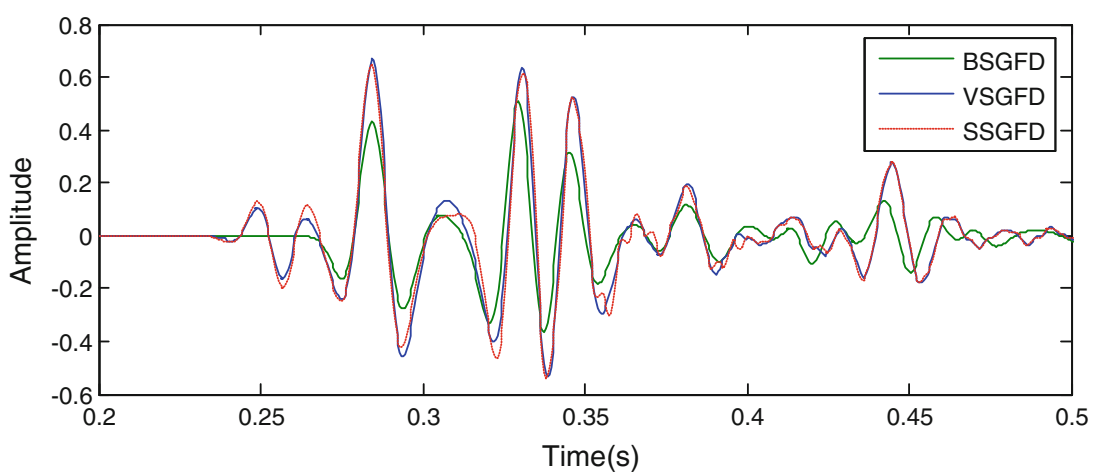

Fig. $9 Z$ component wave motion of solid phase in porous model with caves [receiver locates at $(800,0)$ ]

Table 3 Parameters of low-velocity reservoir model

\begin{tabular}{llllll}
\hline $\begin{array}{l}\text { Model } \\
\text { parameters }\end{array}$ & $\begin{array}{l}\text { P-wave } \\
\text { velocity } \\
(\mathrm{m} / \mathrm{s})\end{array}$ & $\begin{array}{l}\text { S-wave } \\
\text { velocity } \\
(\mathrm{m} / \mathrm{s})\end{array}$ & $\begin{array}{l}\text { Density } \\
\left(\mathrm{kg} / \mathrm{m}^{3}\right)\end{array}$ & Porosity & $\begin{array}{l}\text { Oil } \\
\text { saturation }\end{array}$ \\
\hline $\begin{array}{l}\text { First layer } \\
\text { Second } \\
\text { layer }\end{array}$ & 5,500 & 2,605 & 2,425 & 0 & 0 \\
$\begin{array}{c}\text { Low- } \\
\text { velocity } \\
\text { reservoir }\end{array}$ & 2,100 & 1,212 & 2,200 & 0.15 & 0.5 \\
\hline
\end{tabular}

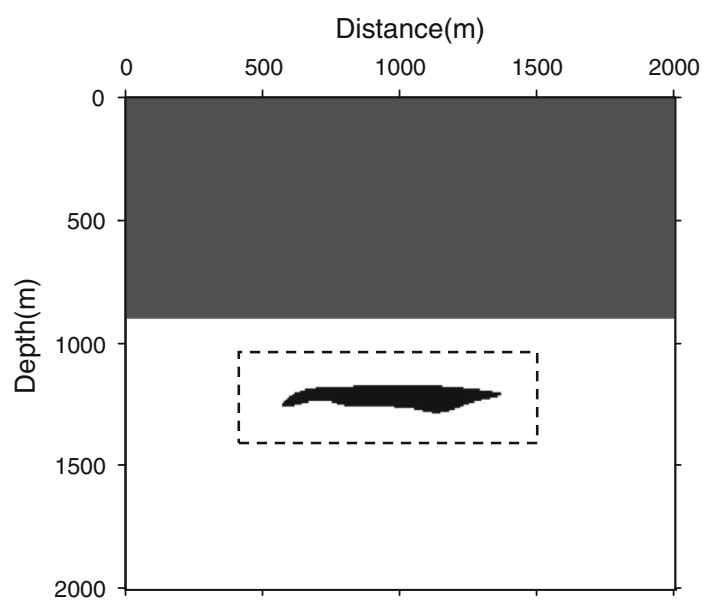

Fig. 10 Porous model with caves

improved. Simulation with small grid-size $(2 \mathrm{~m})$ oversamples the Surrounding high-velocity media and has higher requirements for computer performance while VSGFD method overcomes this limitation. The results of VSGFD method have negligible differences with results of SSGFD method.

Figure 15a shows the $Z$ component shot records simulated for conventional solid single-phase media (using the velocities and densities in Table 3) using the same VSGFD method. Compared with Fig. 12b, we can found that, for the horizontal interface, reflected waves based on porous media theory and single-phase medium theory have reasonable concordance, this is because the first and second layer are designed to be ideal solid phase media (porosity and oil saturation is zero) and can be equivalent to single phase layers. Inside the reservoir, the relative movements between solid and fluid give rise to energy losses, result in attenuation with varying degrees of elastic waves with different frequencies.

\section{Discussion and conclusions}

A velocity-stress, staggered-grid finite-difference scheme with variable grid-size and time-step has been implemented successfully to simulate wave propagation in heterogeneous, porous media. Variable spatial grid-size is chosen to insure sufficient sampling of structures at different scales or layers with high velocity contrasts, time-step is determined adaptively according to grid-size and highest velocity by stability condition of the finite-difference scheme. The proposed method can improve simulation efficiency while insure the accuracy and is more flexible to adapt to parameter changes in formation models.

Forward modeling based on poroelastic theory is more reasonable to simulate the influence of rock porosity and fluid properties on seismic wavefield, however there are still some issues need to be dealt with for practical application, including calculation of elastic parameters $A, N, Q$, $R, \rho_{11}, \rho_{22}$, and $\rho_{12}$, loading of source, treatment of different scales of heterogeneous bodies, the analysis of the complex wavefield, etc. This paper provides an idea to simulate elastic wave propagation and seismic responses while considering the properties of rock pores and fluid in heterogeneous porous media while intensive studies are still needed, especially in low-velocity oil/gas reservoirs with small structures. 


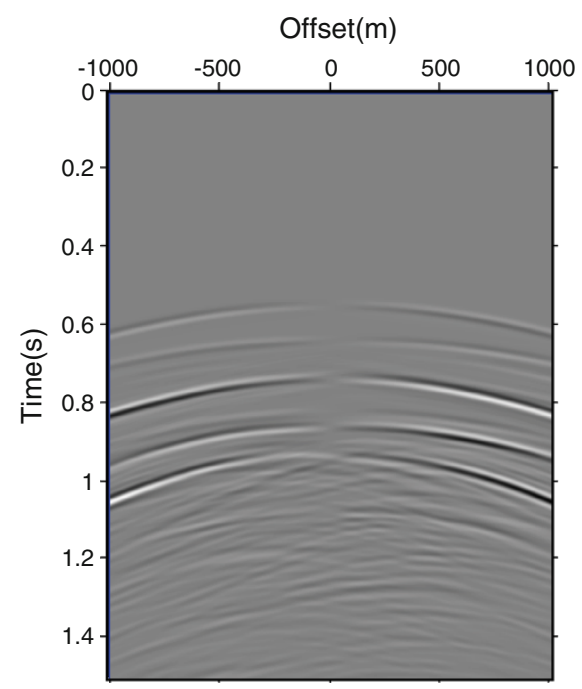

(a) BSGFD

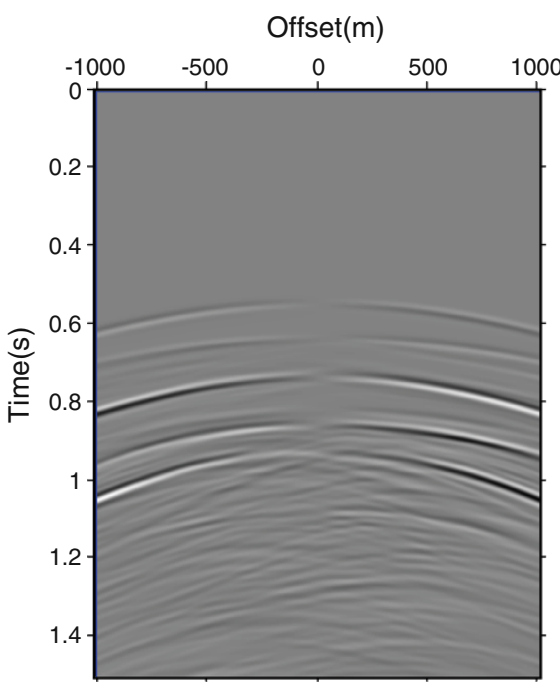

(b) VSGFD

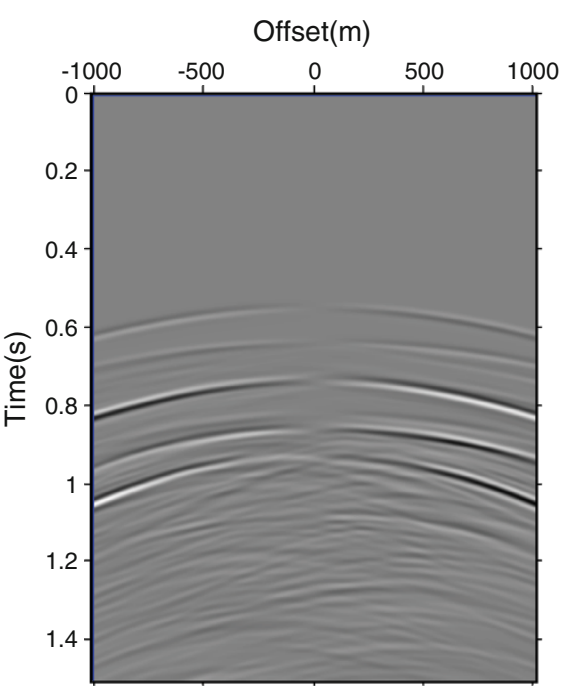

(c) SSGFD

Fig. $11 X$ component shot records of solid phase in porous model with low-velocity reservoir

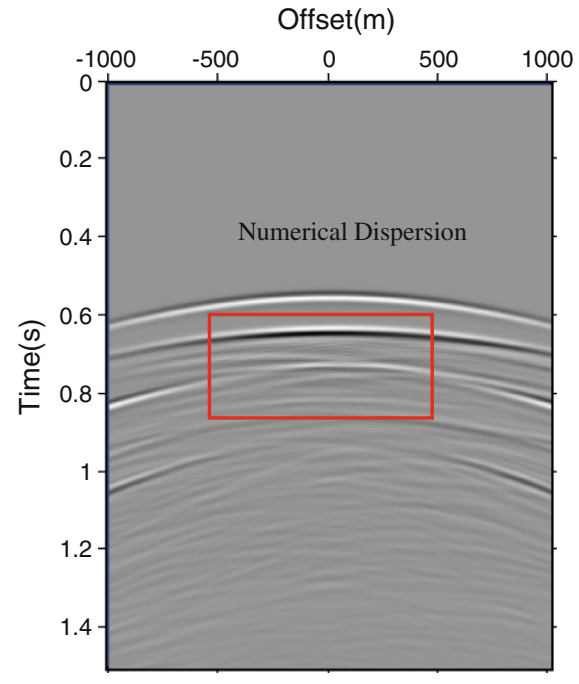

(a) BSGFD

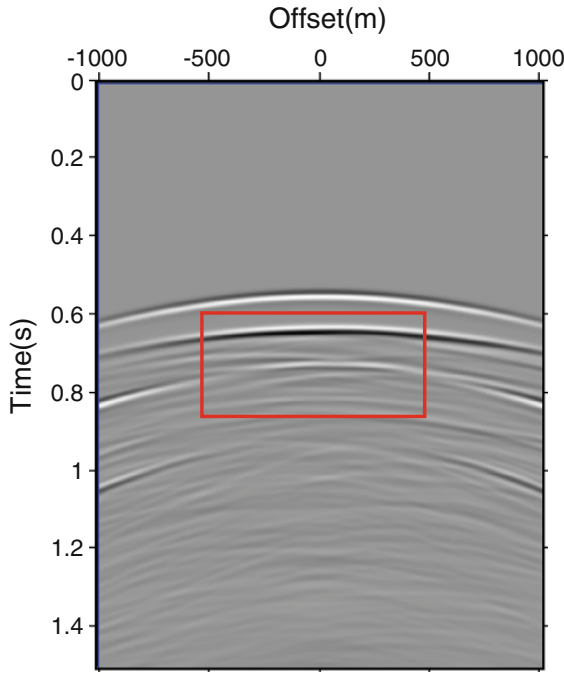

(b) VSGFD

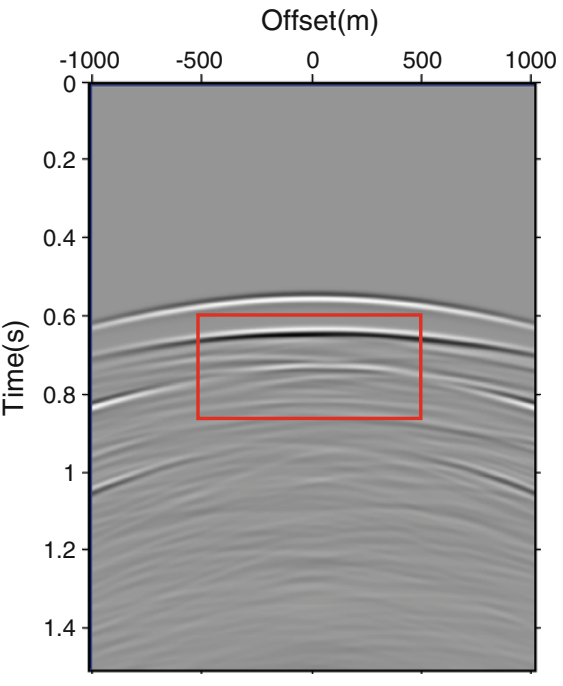

(c) SSGFD

Fig. $12 Z$ component shot records of solid phase in porous model with low-velocity reservoir

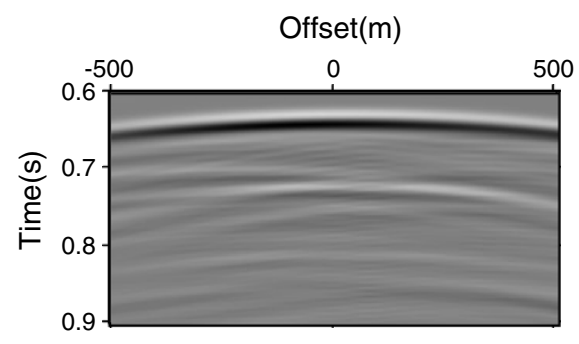

(a) BSGFD

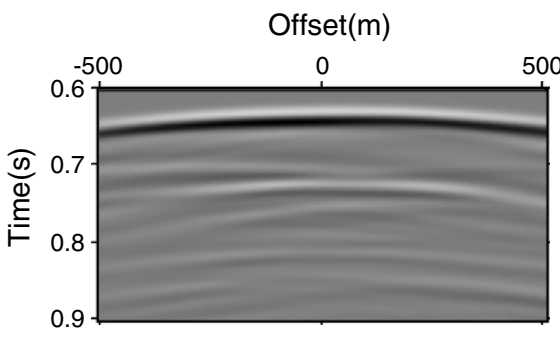

(b) VSGFD

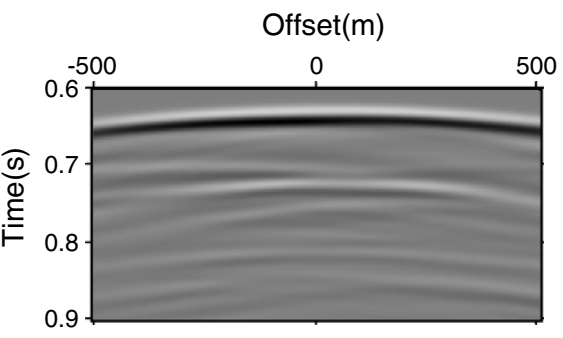

(c) SSGFD

Fig. 13 Enlarged figures of shot records inside dashed rectangle of Fig. 12 


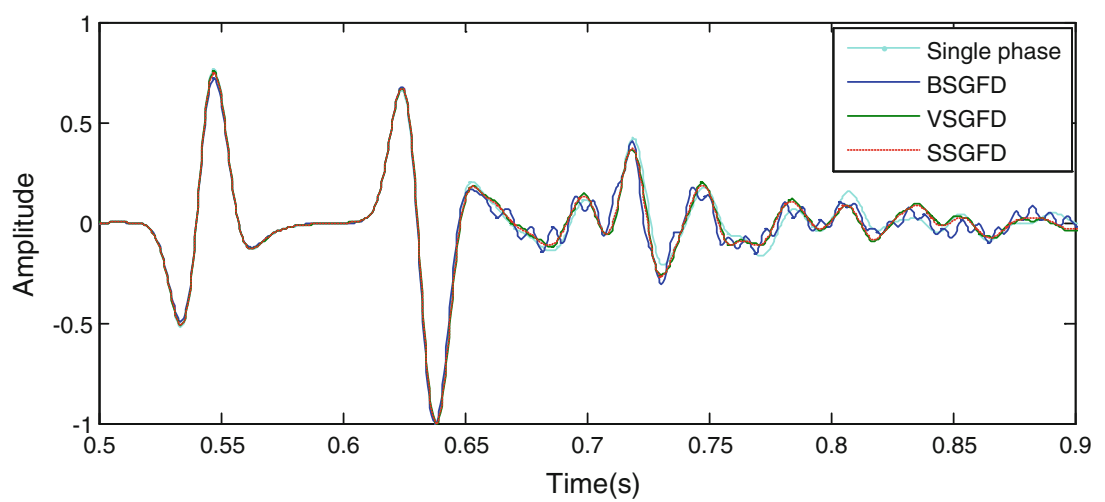

Fig. $14 Z$ component wave motion of Figs. 14a and 12a-c [receiver locates at $(1000,0)$ ]

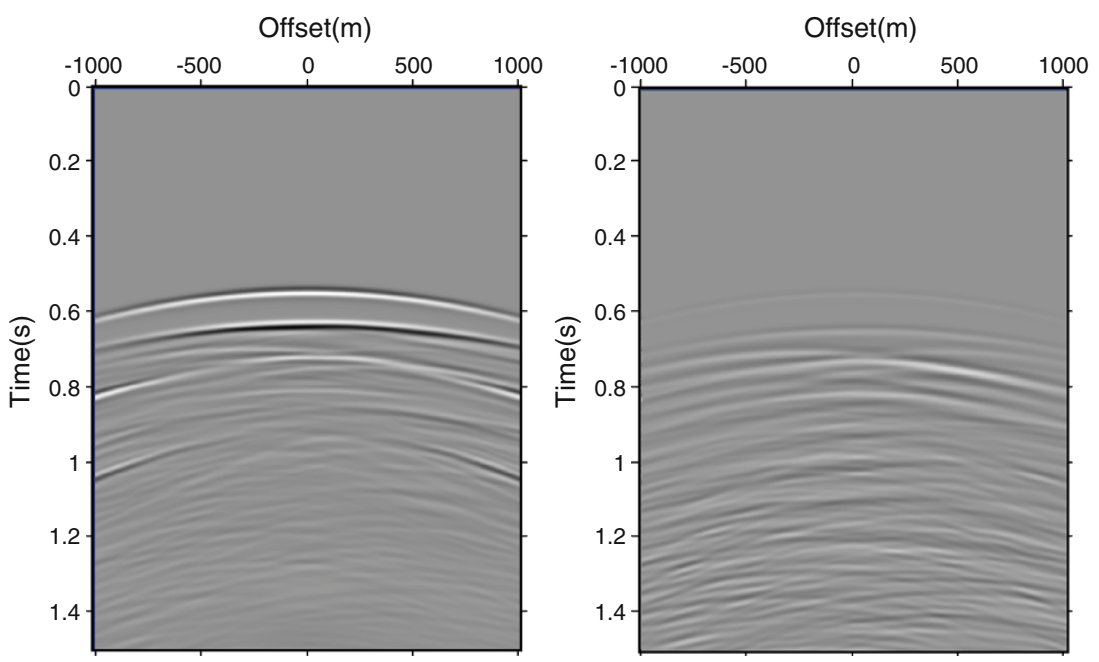

Fig. $15 Z$ component shot records for single-phase media and the difference between Fig. 12a and Fig. 12b

Acknowledgments This study is jointly supported by the National Basic Research Program of China (No. 2013CB228604), the National Science and Technology Major Project (No. 2011ZX05030-004-002, 2011ZX05019-003) and the National Natural Science Foundation (No. 41004050).

\section{References}

Berryman JG (1980) Confirmation of Biot's theory. Appl Phys Lett 37(4):382-384

Biot MA (1956) Theory of propagation of elastic waves in fluidsaturated porous solid, I. Low-frequency range. J Acoust Soc Am 28:169-178

Biot MA (1962) Mechanics of deformation and acoustic propagation in porous media. J Appl Phys 33(4):1482-1498

Carcione JM, Helle HB (1999) Numerical solution of the poroviscoelastic wave equation on a staggered mesh. J Comput Phys 154:520-527

Carcione JM, Quiroga-Goode G (1995) Some aspects of the physics and numerical modeling of Biot compressional waves. J Comput Acoust 3(4):261-280
Dai N, Vafidis A, Kanasewich ER (1995) Wave propagation in heterogeneous, porous media: a velocity-stress, finite-difference method. Geophysics 60(2):327-340

Falk J, Tessmer E, Gajewski D (1998) Efficient finite-difference modeling of seismic waves using locally adjustable time steps. Geophys Prospect 46(6):603-616

Geertsma J, Smit DC (1961) Some aspects of elastic wave propagation in fluid-saturated porous solids. Geophysics 26(1):169-181

Guilbot J, Khaidukov VG, Landa E, Reshetova GV, Tcheverda VA (2008) Finite-difference simulation of elastic waves propagation in multiscale media on the base of local grid refinement. SEG Annual Meeting, pp 2062-2066

Hassanzadeh S (1991) Acoustic modeling in fluid-saturated porous media. Geophysics 56:424-435

Hastings FD, Schneider JB, Broschat SL (1996) Application of the perfectly matched layer (PML) absorbing boundary condition to elastic wave propagation. J Acoust Soc Am 100:3061-3069

Hayashi K, Burns DR, Toksöz MN (2001) Discontinuous-grid finitedifference seismic modeling including surface topography. Bull Seismol Soc Am 91(6):1750-1764

He Y (2008) High-order finite-difference forward modeling of elasticwave in orthorhombic anisotropic media. Master Dissertation, China University of Petroleum (East China), Dongying, pp 1-108 
Huang C, Dong LG (2009) Staggered-grid high-order finite-difference method in elastic wave simulation with variable grids and local time-steps. Chin J Geophys 52(11):2870-2878 (in Chinese with English abstract)

Jastram C, Tessmer E (1994) Elastic modeling on a grid with vertically varying spacing. Geophys Prospect $42: 357-370$

Kang TS, Baag CE (2004) Finite-difference seismic simulation combining discontinuous grids with locally variable time steps. Bull Seismol Soc Am 94(1):207-219

Li ZC, Zhang H, Zhang H (2008) Variable-grid high-order finitedifference numeric simulation of first-order elastic wave equation. OGP 43(6):711-716

Moczo P (1989) Finite-difference technique for SH waves in 2-D media using irregular grids: application to the seismic response problem. Geophys J Int 99:321-329

Pitarka A (1999) 3D elastic finite-difference modeling of seismic motion using staggered grids with nonuniform spacing. Bull Seismol Soc Am 89:54-68

Santos JE, Ravazzoli CL, Carcione JM (2004) A model for wave propagation in a composite solid matrix saturated by a singlephase fluid. J Acoust Soc Am 115:2749-2760

Sun CY, Ding YC (2012) Accuracy analysis of wave equation finite difference with dual-variable algorithm. OGP 47(4):545-551
Sun LJ, Yin XY (2011) A finite difference scheme based on PML boundary condition with high power grid step variation. Chin $\mathbf{J}$ Geophys 54(6):1614-1623 (in Chinese with English abstract)

Tessmer E (2000) Seismic finite-difference modeling with spatially varying time steps. Geophysics 65(4):1290-1293

Wang Y, Xu J, Schuster GT (2001) Viscoelastic wave simulation in basins by a variable-grid finite-difference method. Bull Seismol Soc Am 91(6): 1741-1749

Wenzlau F, Müller TM (2009) Finite-difference modeling of wave propagation and diffusion in poroelastic media. Geophysics 74(4):T55-T66

Wu GC (2006) Seismic wave propagation and imaging in anisotropy media. China University of Petroleum Press, Dongying, pp 1-292 (in Chinese)

Zhang HL, Wang XM, Zhang BX (2004) Acoustic field and wave in borehole. Science Press, Beijing, pp 1-263 (in Chinese)

Zhu X, McMechan GA (1991) Numerical simulation of seismic responses of poroelastic reservoirs using Biot theory. Geophysics 56(3):328-339

Zhu SW, Qu SL, Wei XC, Liu CY (2007) Numeric simulation by grid-various finite-difference elastic wave equation. OGP 42(6):634-639 